\title{
Superconductivity and their Applications
}

\author{
A. Roque ${ }^{1,2}$, D. M. Sousa ${ }^{2,3}$, V. Fernão Pires ${ }^{1,2}$, E. Margato ${ }^{4}$ \\ ${ }^{1}$ Department of Electrical Engineering \\ ESTSetúbal/Instituto Politécnico de Setúbal \\ Campus of IPS, Estefanilha, 2914-761 Setúbal, Portugal \\ Phone/Fax number:+351 265790000; antonio.roque@estsetubal.ips.pt, vitor.pires@estsetubal.ips.pt

\begin{abstract}
${ }^{2}$ INESC-ID
Av. Alves Redol 9, 1000-029 Lisboa, Portugal

Phone/Fax number:+351 213100300/+351 213100235 Lisboa, Portugal

${ }^{3}$ DEEC AC-Energia, Instituto Superior Técnico, Universidade de Lisboa

Av. Rovisco Pais, 1 - 1049-001 Lisboa, Portugal, Phone/Fax number: +351 218417429/+351 218417167, duarte.sousa@ist.utl.pt
\end{abstract}

\begin{abstract}
${ }^{4}$ CEEI, ISEL-Instituo Superior de Engenharia de Lisboa, Instituto Politécnico de Lisboa, and INESC-ID, Av. Rua Conselheiro Emídio Navarro 19059-007 Lisboa. Portugal, Phone/Fax number: +35121 8417429/+351218417167, efmargato@isel.ipl.pt
\end{abstract}

\begin{abstract}
The research in the field of superconductivity has led to the synthesis of superconducting materials with features that allow you to expand the applicability of this kind of materials. Among the superconducting materials characteristics, the critical temperature of the superconductor is framing the range and type of industrial applications that can benefit from them. Some examples of industrial applications incorporating superconducting materials stand out in this paper. Among other possibilities, the nuclear magnetic resonance, the magnetic levitation train, the transport processing of electrical energy (motors, generators, transformers and power lines) and superconducting magnetic energy storage (SMES) systems are already solutions contributing to the nowadays daily life, but more than that, are solutions that will contribute to improve the quality of life of many human beings in the near future. In addition to these solutions, in this paper are presented and discussed the pros and cons of a solution designed for the fast field cycling nuclear magnetic resonance technique that benefits of the usage of superconducting blocks.
\end{abstract}

\section{Key words}

Superconductivity; Energy storage; Superconducting applications; Nuclear magnetic resonance (NMR).

\section{Introduction}

Superconducting is now starting to be an interesting technology to be applied to real applications. This technology is based in some peculiar effects. In fact, in a classical superconductor the resistance goes to zero when the temperature becomes higher than the absolute zero, which is known as the critical temperature. In 1908 the Dutch physicist Heike Onnes Kamerling began to work in the field of low temperature physics through liquefying helium. Three years later, in 1911, he found that below 4.2K the resistivity of mercury was null. Onnes also found that the application of a magnetic field causes a decrease in the critical temperature, $T_{c}$. Subjecting the superconductor to a sufficiently intense field, the superconductivity disappears and the material changes to its normal state. Being the temperature a thermodynamic decisive variable for the occurrence of the transition of phase of the normal state to the superconducting state, it is not unique, since the intensity of the magnetic field also causes phase transition of that occurrence. This means that when the material is in the superconducting state and is applied a magnetic field whit a value behind to the critical value of the magnetic field intensity, $H_{c}$, the superconductivity is destroyed and the material has a transition of phase, namely changes from the superconducting state to normal conduction state.

\section{Meissner Effect}

German physicians Walther Meissner and Robert Oschsenfeld verify in 1933 that superconductors present different properties from the ideal conductors. By submitting the tin $\mathrm{Sn}$, and lead $\mathrm{Pb}$, at temperatures below the critical temperature and under the action of a magnetic field, they have found that magnetic field lines do not penetrate the sample of these materials, which corresponds to $\vec{B}=0$ within the sample, being this phenomenon known as the Meissner effect [1]. With this discovery it was demonstrated that a superconductor is a diamagnetic material, while an ideal conductor despite its zero resistivity does not have this property. In this way, it was defined a new state of matter with very particular properties.

\section{Main characteristics of superconductors}

The diagram of Fig. 1 summarizes the phenomena associated with the main characteristics of superconductors (SC): diamagnetism and zero resistivity. Due to the mentioned characteristics, superconducting 
materials can withstand high currents which are called by supercurrents. It was also verified that the material loses its superconducting properties when the critical magnetic induction or critical temperature is exceeded.

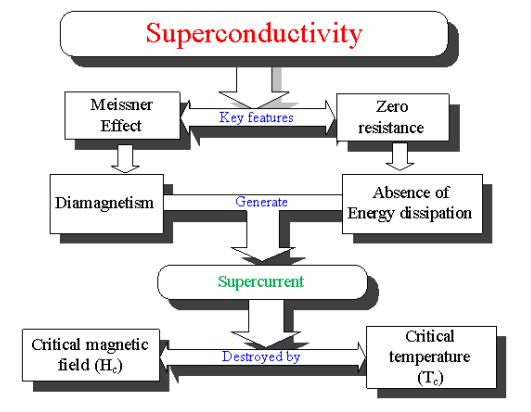

Fig. 1. Diagram of the main characteristics of SC

\section{Classification of superconductors}

The response of the superconductors to the application of an external magnetic induction led to the classification of the superconductor material into two types: Type I and Type II superconductors. The superconductors of Type $I$ are generally metals and some metallic alloys, being generally electrical conductors at ambient temperature, and acquires superconductive properties at very low temperatures. The first element to be discovered by physical Heike Onnes in 1911 , was the mercury $(\mathrm{Hg})$, which had a virtually zero resistivity when subjected to a temperature of $4.2 \mathrm{~K}$.

Type II superconductors are formed essentially by metallic alloys and other compounds. The exceptions are the pure metals Vanadium (V), Technetium $(\mathrm{Tc})$, niobium $(\mathrm{Nb})$ and carbon (C) that are Type II superconductors.

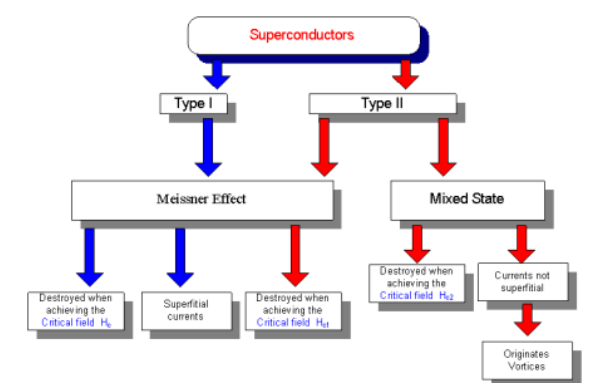

Fig. 2. Diagram of the classification of superconductors.

In general the critical temperature associated with this type of superconductors is much higher than that of Type I. The first discovered superconducting material of Type II was an alloy of lead and bismuth manufactured in 1930 by W. Haas and J. Voogd [2], which verified that the alloy present different characteristics of the superconductors Type I. The diagram presented in Fig. 2 shows a summary of the classification of superconductors.

\section{Ginzburg-Landau Theory}

In 1950, Soviet physicists Landau and Ginzburg formulated a theory that explains the transition thermodynamic properties of the normal state to the superconducting state, using quantum mechanics to describe the effect of the magnetic field.
The first Ginzburg-Landau theory refers to the intuitive idea that a superconductor contains a supercurrent density $\vec{J}_{s}$ and a normal current density $\vec{J}_{n}$, being the total current density given by: $\vec{J}_{t}=\vec{J}_{n}+\vec{J}_{s}$. The density of the supercurrent can be described by a wave function $\Psi$ given by equation (1).

$$
\left|\Psi^{2}\right|
$$

This wave function $\Psi$ decreases when close to the critical temperature, since the number of superelectrons decreases with the increase of the temperature. Thus a decrease in the number of superelectrons implies a reduction of the wave function, according equation (1).

The wave function, $\Psi$, in the superconducting state is different from zero, while in the normal state the wave function is zero, as expression (2).

$$
\left\{\begin{array}{l}
\psi=0 \text { se } T>T_{c} \\
\psi \neq 0 \text { se } T<T_{c}
\end{array}\right.
$$

The Ginzburg-Landau theory also provides the existence of another fundamental value related to superconductivity, which is designated as coherence length, and that can be estimated by equation (3).

$$
\xi^{2}=\frac{h^{2}}{m^{*}|\alpha|}
$$

Where $h$ is the Planck constant, $m^{*}$ the mass of a pair of electron and $\alpha$ a parameter phenomenological depending on the temperature.

The equation that relates the London penetration length with the wave function is given by:

$$
\lambda_{L}^{2}=\frac{m^{*}}{\mu_{0}\left|\Psi^{2}\right| e^{*^{2}}}
$$

The ratio between the two characteristic lengths, London penetration length and coherence length, defines the Ginzburg-Landau parameter given by equation (5).

$$
k=\frac{\lambda_{L}}{\xi}
$$

If the value of $k$ is less than $1 / \sqrt{ } 2$ then the material is superconductor Type $I$ and for $k$ greater than $1 / \sqrt{ } 2$ the material is superconductor Type II [3].

As can be seen from the Fig. 3 a), the London penetration length is much lesser than the coherence length.

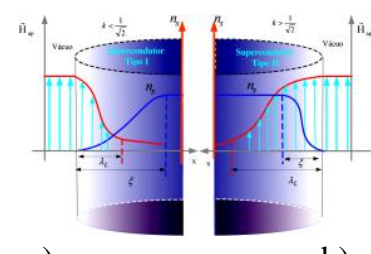

a)

b)

Fig. 3. Evolution of the magnetic field and superconducting electrons inside of a superconducting sample: a)Type I, b) Type II

In Fig. 3. b) it can be seen that the evolution of the decay of the magnetic field intensity inside the sample is slower, contrary to what happened in the Fig. 3 a). In this case, the evolution of the characteristic that represents the superconducting electrons is faster when is analyzed from the superconductor surface to the interior. This behavior results in an increased penetration length and a shorter 
coherence length. When the ratio between the two magnitudes, given by the expression (5) exceeds $\frac{1}{\sqrt{2}}$ then the superconductor is said to be of Type II.

\section{Main Applications}

With the discovery of High Temperature Superconductors (HTS) the commercial applications involving these materials became very interesting. In fact, the high power density and electrical efficiency of superconductor wire results in highly compact, powerful devices and systems that are more reliable, efficient, and environmentally benign. Thus, their application involves areas such as, medicine, transportation electrical grids and industry.

\section{A Magnetic Levitation Train}

One of the main applications of the superconductivity is in transportation, more specifically in trains through the magnetic levitation. Due to this, the friction between the train and the rail is eliminated. As a result, these trains can achieve high speed with lower consumption and noise. The maglev train in Shanghai that began commercial operations in 2004 is one example of a real application where this technology is used (Fig. 4). That line has a distance around $30 \mathrm{~km}$ between the financial area of Xangai and the airport (Pudong). It can achieve $450 \mathrm{~km} / \mathrm{h}$ with a time travel of 8 minutes. Another commercial maglev train is the one that was built in Changsha, China. This train is the first one fully developed in China, being the first mid-low speed maglev in China, and with the purpose of be an experimental line. The line stretches 18.5 kilometers and links the Changsha South Railway Station with the local airport, being the longest one of its kind in the world. It was open in May 2016.

However, it is expected the implementation of new projects with this technology. The Japanese rail operator announced the implementation of a new magnetic levitation train able to achieve $600 \mathrm{~km} / \mathrm{h}$. They expect to have in operation this new train in 2027 [4].This line will have a $286 \mathrm{~km}$ route with an expected time travel of 40min less than an hour compared with the same route travelled by the actual "shinkansen" or bullet trains.

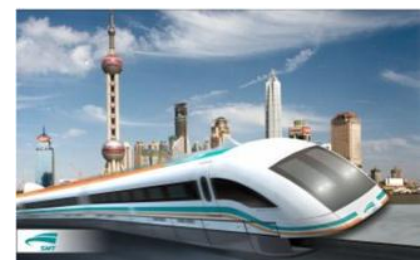

Fig. 4. Maglev train at Xangai.

\section{B Transmission and Distribution Electrical Systems}

The use of superconductivity for the electricity transport is another area where this technology can be used with several benefits. One of the main advantages is the lack of resistive losses improving in this way the efficiency of the energy transportation, [5]. However, there are other advantages such as, higher transport capability, smaller overall size leading to lower impact on soil, possibility of using exiting corridors and lower impact on nature. Due to this, in the last years it started the use of semiconductors in electricity transport. Some examples of that application can be seen in table 1 , where it is possible to verify that this technology was used in medium and high voltage transportation [6].

Table I. - Type Sizes

\begin{tabular}{|c|c|c|c|}
\hline Year & Local & Length [m] & Capacity [MVA] \\
\hline 2008 & $\begin{array}{l}\text { USA (Long } \\
\text { Island) }\end{array}$ & 600 & $574(138 \mathrm{kV} \mathrm{AC}, 2.4 \mathrm{kA})$ \\
\hline 2014 & Germany (Essen) & 1000 & $40(10 \mathrm{kV}$ AC, $2.3 \mathrm{kA})$ \\
\hline $\begin{array}{c}\text { Under } \\
\text { construc. }\end{array}$ & $\begin{array}{l}\text { Netherlands } \\
\text { (Amsterdam) }\end{array}$ & 6000 & $250(50 \mathrm{kV} \mathrm{AC})$ \\
\hline 2015 & $\begin{array}{c}\text { Russia (St. } \\
\text { Petersburgo) }\end{array}$ & 2500 & $50(20 \mathrm{kV} \mathrm{DC}, 2.5 \mathrm{kA})$ \\
\hline 2014 & Japan (Ishikari) & 2000 & $100(710 \mathrm{kV} \mathrm{DC}, 5 \mathrm{kA})$ \\
\hline 2013 & Corea (Icheon) & 100 & $154(154 \mathrm{kVAC}, 3.75 \mathrm{kA})$ \\
\hline 2015 & $\begin{array}{c}\text { Corea (Jeju } \\
\text { Island) }\end{array}$ & 1000 & $154(154 \mathrm{kVAC}, 3.75 \mathrm{kA})$ \\
\hline 2014 & Corea (Jeju) & 500 & $500(80 \mathrm{kV} \mathrm{DC})$ \\
\hline 2014 & $\begin{array}{c}\text { USA } \\
\text { (Westchester) }\end{array}$ & 170 & $96(13.8 \mathrm{kV} \mathrm{AC} / 4 \mathrm{kA})$ \\
\hline 2015 & $\begin{array}{c}\text { Japan } \\
\text { (Yokohama) }\end{array}$ & 250 & $200(66 \mathrm{kV} \mathrm{AC,5kA})$ \\
\hline 2011 & China & 360 & $13(1.3 \mathrm{kV} \mathrm{DC}, 10 \mathrm{kA})$ \\
\hline
\end{tabular}

Another application associated with the electrical transmission and distribution where is used the superconductivity is the fault current limiters. These devices allow to limit short-circuit currents on utility distribution and transmission networks through the use of HTS. Unlike reactors or high-impedance transformers, they allow to limit fault currents without introduce an impedance in the circuit in normal operation. Due to this interesting characteristic, this technology is now being extensively studied in order to be applied in future networks. Some of the main applications are: at the output of a transformer to protect an entire bus; to protect an individual feeder; between two buses that are supplied by a transformer (the two buses are tied, but in a situation of a faulty bus, that bus will receive the full fault current of only one transformer).

\section{Transformers, Generators and Motors}

Besides the use of superconductivity for the electricity transport, it is also possible to use this technology in other electrical power systems. Examples of this are the use of superconductivity in transformers, generators and motors. The interest to use the superconductivity in transformers started in 1960s with the appearance of low-temperature superconductors and their use in the transformer windings. In fact, several manufacturers around the world, among them the European (ABB and Alstom), the Japonese (KEPC) and Americans (Westinghouse) (USA) made some tests with these equipment's. However, with the discovery of HTS materials in 1986, this interest increased since allowed to eliminate the cumbersome cooling devices. Several advantages are associated with HTS transformers, namely, greater efficiency, ability to run above rated power without affecting transformer life, smaller, lighter and quieter. Fig. 5 a) shows the prototype of a single-phase transformer with $2 \mathrm{MVA}(66 \mathrm{kV} / 6.9 \mathrm{kV})$ 
that was developed by a research group in Japan, [7]. Some transformers with HTS materials have been developed and tested. The ABB Company tested a $630 \mathrm{kV}$ three-phase transformer for the Swiss utility to be used for one year under regular operational conditions [8]. Another project was developed by the Siemens Company, where a 1MVA demonstrator transformer for railway applications was tested with success [9].

Through the use of superconductors in generators is possible to obtain several benefices. In the classical generators there are losses associated to the rotor windings and armature bars. However, through the use of superconducting wire for the field windings, these losses can be practically eliminated. On other hand, the fields that are created in the armature by the rotor are not limited by the saturation characteristics of the iron and the armatures are constructed without iron teeth. Due to this, it is possible to eliminate the losses experienced in the armature teeth. Thus, with the use of HTS material many research was done in order to become a reality the use of these generators. Besides the advantages described before, generators with HTS technology can provide fast response and reactive power support. Due to this, HTS generators can be used to support the power grid in order to keep running smoothly in the face of new patterns of power flows being brought on by the deregulation of power generation throughout the world. Since HTS generators allows to reduce their volume when compared with classical generators, their use in ships is very attractive. In fact, the US Navy are interested in use these motors since their new generation of surface ships is based on electrical drive due to lower operating and support cost [10]. Under this context, a 3.7MW engine has been tested A generator with $450 \mathrm{~kW}$ was also developed by the Siemens Company. Another project by Siemens, is a 4MW propulsion motor [9].

As in the case of the generators, the use of the superconductivity technology was also tested in electrical motors. As in the case of the generators, the interest in using this technology was increased with the use of the HTS materials. In fact, through the replacement of the conventional copper coils by superconducting wires, it is possible to develop motors more energy efficient, less expensive, with reduced size and weight, but also removing a significant source of motor noise.

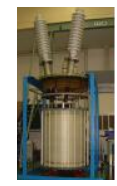

a)

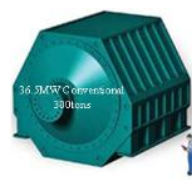

b)

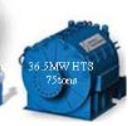

c)
Fig. 5. a) Prototype 2 MV A HTS transformer [7],

b) Conventional Machine c) HTS Machine [11].

The use of this technology will have advantages particularly for large industrial applications. In Fig. 5 b) is shown a classical and a HTS motor. From the Fig, 5 c) is possible to verify that the size of the HTS motor is much smaller than the classical one.

Due to the advantages of the application of this technology in motors some machines have been developed. One of the examples is the $36.5 \mathrm{MW}$ high-temperature superconductor
(HTS) ship propulsion motor developed by the American Superconductor Corp. (AMSC) and Northrop Grumman Corp. in 2009 .Through the incorporation of coils with HTS wire, it is possible to carry 150 times the current of similar-sized copper wire, making the motor less than half the size of conventional motors used on the first two DDG1000 hulls and with a reduce ship weight by nearly 200 metric tons, [11] [12]. This will make new ships more fuelefficient, saving close to $\$ 1$ million annually, depending of the cost of oil, and free up space for additional warfighting capability, as AMSC said. Sumitomo Electric and a Japanese research group succeeded to develop a HTS ship motor cooled by liquid Nitrogen (Fig. 6 a). This motor has a nominal power of $365 \mathrm{~kW}$ with a speed of $250 \mathrm{rpm}$ and weighing 4.4ton. This company also developed a motor with around $30 \mathrm{~kW}$ power and $120 \mathrm{Nm}$ torque. They developed this small superconducting motor and successfully demonstrated for an electrical passenger car (Fig. 6 b). By these examples it was demonstrated that superconducting motors will enable to get larger torque, reduce the space and get higher energy efficiency, [13].

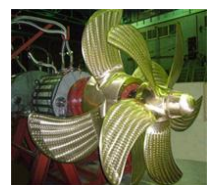

a)

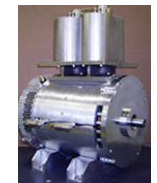

b)
Fig. 6. Superconducting motors Sumitomo: a) Contrahelical propulsion system b) electrical passenger car [13].

\section{Energy Storage}

There are several technologies that can be used to store electricity from the grid. One of the technologies is based on the magnetic energy storage. However, in order to increase the capacity of these systems, it was proposed the use of the superconductivity. In fact, these systems designated by Superconducting Magnetic Energy Storage (SMES) are based on a magnetic field in a coil comprised of superconducting wire with near-zero loss of energy. These devices are typically consisted by a cryogenically cooled superconducting coil connected to the electrical source through a power condition system.

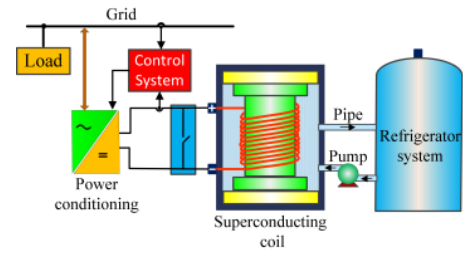

Fig. 7. Typical scheme of a Superconducting Magnetic Energy Storage (SMES).

Fig.7 shows a typical scheme of this type of application. It consists in a DC/AC power convert that controls the power that will be transferred to the superconducting coil, and vice-versa. At the terminals of the superconducting coil there is a switch that turns on when the system is in stand-off (allowing to maintain the stored energy in the superconducting coil). For low temperature materials it is used in the refrigerator system liquid helium while for high temperature materials is used nitrogen. 
SMES are characterized by their capability of store or discharge in an almost instantaneous way large quantity of power. Due to this, they are an important device in high power applications such as in transmission and distribution electrical systems. In these applications, they can play an important role in the grid reliability since it allow to alleviate congested power lines and attenuate the impact of high penetration of renewable energy sources. Besides that, they are also characterized by the capability to improve power quality of critical loads, present less environment impact when compared with other storage systems such as batteries and long term duration, [14]. Another storage technology where superconductivity can be used is in flywheels, [15]. These storage systems are based on rotating electrical machines. In these systems, the stored energy is directly proportional to the mass of the object in movement but proportional to the square of their rotational speed, [16]. According to this there are two types of devices, the low speed and the fast speed flywheels. Most of the commercial flywheels are low speed and made of metal with a very huge size. However, many works have been done in order to reduce the size of the flywheel through the use of high speeds. In these flywheels instead of use metal it is employed fiber-reinforced plastics (FRP). Thus, with the use of these materials is possible to obtain higher operational speeds since they have very high strength in the radial direction. The main problem of this technology is the bearings. Besides the limits in the development of mechanical bearings with contact between the stationary and rotating parts they also generate enough loss to render the system uneconomical. In order to solve this problem a non-contact active magnetic bearing can be used. However this type of bearing consumes power, which is dissipated as heat in the copper electromagnets. To overcome this problem it can be used superconducting magnetic bearings. With this type of technology an overall one-day, "roundtrip" system efficiency of $84 \%$ can be achieved, which is an acceptable value, [17-18].

\section{E - Nuclear Magnetic Resonance}

The Nuclear Magnetic Resonance (NMR) is a powerful spectroscopic technique and widely used in various fields of basic or applied research in the areas of Physics, Chemistry, Materials Science, Medicine and Biology, among others. However, some of the conventional spectroscopic techniques do not allow to obtain results in good experimental conditions when the amplitude $\vec{B}=0$ is below certain values, corresponding to frequencies between $\cong 0$ and $\cong 0.2 \mathrm{~T}$ for the "spin" of the ${ }^{1} \mathrm{H}$ proton, which gave rise to the NMR technique Field Cyclical Fast. In this paper is given a global overview of the conventional NMR and the Fast Field Cycling NMR technique, both having solutions based on superconducting materials.

\section{E.1 RMN Conventional}

One of the major commercial applications of superconductivity is in the medical diagnostic. In fact, images obtained from nuclear magnetic resonance, has now an important role in the context of the image diagnose. This technology is based on the generation of an intense magnetic field that is able to influence the orientation of the atoms nuclei that constitute the human body. In order to obtain high-resolution images, it is used superconducting coils capable of generate strong magnetic fields (Fig. 8). The protons of the hydrogen atoms that are present in water and fat molecules can be considered as small magnets. When the human body is subjected to a magnetic field those small magnets (protons) will be oriented by that magnetic field. When the magnetic field ceases those small magnets will return to its equilibrium position through the emission of an electrical signal. That electrical signal will depend on the type of the body tissue. If the tissue is fat that means that the matter is very dense and the relaxation time of protons will be short leading to a dark spot in the image diagnosis.

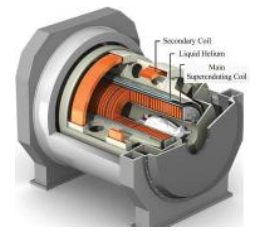

Fig. 8. Nuclear Magnetic Resonance equipment [19].

If the tissue is less dense then the relaxation time of protons is higher and will give a clear spot on diagnostic imaging.

The diagnosis through this type of images has now become an essential medical procedure, due mainly to the development of the capability of the computers processing, since is required an analysis of large amount of data that is generated during the examinations.

\section{E.2. Fast Field Cycling - Nulear Magnetic Resonance}

A fast field cycling (FFC) nuclear magnetic resonance relaxometer is basically composed of the following components: a magnet; a power supply that provides power to the magnet; a RF circuit; a receiver that processes the NMR signals; and a console (Fig. 9.a).

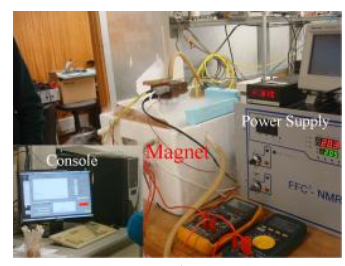

a)

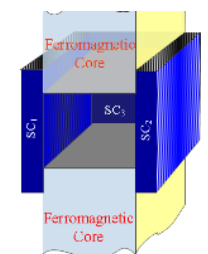

b)
Fig. 9.a) Magnet, console and power supply.

b) Magnet with superconducters $\left(\mathrm{SC}_{1}, \mathrm{SC}_{2}, \mathrm{SC}_{3}\right)$

With this technique a sample (from liquid crystals to polymers) is submitted to a given magnetic induction (Zeeman induction) depending on the type of particle under analysis.

One possibility for creating the magnetic induction is through Helmoltz coils [20]. Different solutions based on this configuration has been developed targeting different magnetic induction homogeneities. At the end, it is possible to design coils with distinct characteristics [21]. In addition, to achieve the target magnetic induction required by the FFC technique with Helmoltz coils a high current is required. This current is in general associated with large power losses in the coil due to the Joule effect, and therefore, a complex cooling system is required. 
To overcome these technical issues, a magnet was developed based on a magnetic circuit. With this solution, the magnet current was substantially reduced (5 times smaller for the same value of magnetic induction), and thereby, increasing the system efficiency accompanied by a reduction of volume and weight of the relaxometer. After validating this type of solution, the next step was to increase the homogeneity of the magnetic induction in the air gap and to reduce the losses in the magnetic circuit [22]. This was accomplished using superconducting blocks (Bi-2223), Fig. 9.b), which due to its diamagnetic properties will allow to obtain a more uniform magnetic induction in the section where the sample is inserted, as illustrated in Fig. 10. Comparing the magnetic induction distribution in the magnet without and with the effect of the superconducting parts, Fig. 10. a) and Fig. 10.b), respectively.

This solution allowed to reduce the losses by Joule effect in the magnet, because the magnet resistance decreases when immersed in liquid nitrogen and, so that, requiring less current to get the target magnetic induction, which must be $0.2081 \mathrm{~T}$

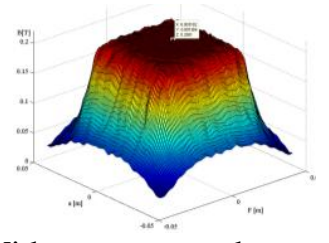

a) Without superconductors

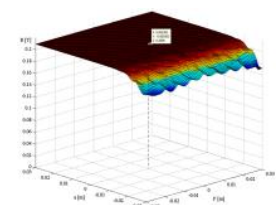

b) With superconductors
Fig. 10. Magnetic induction distribution.

\section{Conclusion}

In this paper was pointed out the relevance of the superconducting materials in nowadays applications. Framed by the critical temperatures of the superconducting solutions, the range and type of industrial applications that can benefit from them has been increasing during the last decade.

As core examples, the nuclear magnetic resonance, the magnetic levitation train, the transport processing of electrical energy (motors, generators, transformers and power lines) and superconducting magnetic energy storage (SMES) systems are solutions that take advantage of the superconductor's characteristics. In addition to these applications, new ones has been developed incorporating superconducting parts.

A magnet of a fast field cycling nuclear magnetic resonance equipment was designed considering the usage of superconducting blocks. As described, the features of this new application improved, benefiting of the usage of superconducting materials being expected that, in a near future, much more applications will be designed and developed using superconducting parts.

\section{Acknowledgement}

This work was supported by national funds through Instituto Politécnico de Setúbal and Fundação para a Ciência e a Tecnologia (FCT) with reference UID/CEC/50021/2013.

\section{References}

[1] W. Meissner; R. Oschsenfeld: "Ein neuer Effect bei Eintritt der Supraleitfahigkeit", Die Naturwissenschaften, vol. 21 (44), pp. 787 788, 1933.

[2] P. Branício: "Introdução à supercondutividade, Suas Aplicações e Mini-Revolução Provocada Pela Redescoberta do MgB2: Uma abordagem Didática", Revista Brasileira de Ensino de Física, vol. $23, n^{\circ} 4,2001$

[3] A. Abricosov: "On the Magnetic Proprieties of Superconductors of the Second Group", Sov. Physics JETP, Vol. 5 N. 6, 1957.

[4] S. Nishijima and al.: "Superconductivity and the environment: a Roadmap", Supercond. Sci. Technol. 26, 113001 (35pp), 2013.

[5] H. Jones: "Superconductors in the transmission of electricity and networks", Elsevier, Energy Policy 36 4342-4345, 2008.

[6] H. Thomas and al.: "Superconducting transmission lines - Sustainable electric energy transfer with higher public acceptance?" Elsevier, 2016.

[7] T. Bohno and al.: "Development of $66 \mathrm{kV} / 6.9 \mathrm{kV} 2 \mathrm{MVA}$ prototype HTS power transformer", Physica C: Superconductivity, 426-431, Part 2, pp. 1402-1407, 2005.

[8] H. Zeguer: "630kVA high temperature superconducting transformer", Elsevier, Vol. 38, Issue 11, Pages 1169-1172, 1998.

[9] A. Narlikar: "Higth Temperature Superconductivity 2- Engineering Applications", Springer, ISBN 978-3-642-07369-4, 2004.

[10] M. Tinkham and C. J. Lobb: "Physical Properties of the New Superconductors", Solid State Phys. 42, p. 91, 1988.

[11] American Superconductor Corporation, Annual Report, Form 10-k, Securities and Exchange Commission Washington, D.C. 20549 2005.

[12] [Online]. Available: http://www.powermag.com/ superconductor-motor-for-navy-passes-full-power-test/.

[13] [Online]. Available: http://globalsei.com/super/ magnet_coil_e/evmotor.html.

[14] M. Ali and al: "An Overview of SMES Applications in Power and Energy Systems", IEEE Trans. on Sustainable Energy, Vol. 1, No. 1, 2010.

[15] M. Strasik and al.: "Design, Fabrication, and Test of a 5-kWh/100kW Flywheel Energy Storage Utilizing a High-Temperature Superconducting Bearing", IEEE Transactions on Applied Superconductivity, vol. 17. No. 2, 2007.

[16] H. Ibrahim; A. Ilinca; J. Perron: "Energy storage systemsCharacteristics and comparisons", Elsevier, Renewable and Sustainable Energy Reviews 12 1221-1250, 2008.

[17] X. Xue; K. Cheng; D, Sutanto: "Power System Applications of Superconducting Magnetic Energy Storage Systems”, Fourtieth IAS Annual Meeting, 2005

[18] P. Tixador and al.: "Design and First Tests of a 800kJ HTS SMES, IEEE Trans. on Applied Superconductivity, vol. 17. No. 2, 2007.

[19] [Online]. Available: http://www.clinicaidisa.com.br/artigos/ view/id/42/video-como-um-equipamento-de-ressonanciamagnetica.html\#.

[20] F. Noack: "NMR Field-Cycling Spectroscopy: Principles and Aplications", Progress in NMR Spectroscopy, Vol. 18, pp. 171-276, 1986.

[21] D. M. Sousa; G. Marques: "Study of the Air Gap Magnetic Field Distribution of a Nuclear Magnetic Resonance Iron-Core Magnet”, 1st IEEE Region 8 International Conference on Computational Technologies in Electrical and Electronics Engineering, Novosibirsk - Russia, pp. 242-247, 2008.

[22] A. Roque; D. M. Sousa; E. Margato; V. Machado; P. Sebastião and G. Marques: "Magnetic Flux Density Distribution 3D Analysis in the Air Gap of a Ferromagnetic Core with Superconducting Blocks", IEEE Trans. on Applied Superconductivity, Vol. 25, nº 6, 2015. 